\title{
Laminar boundary-layer separation over a circular cylinder in uniform shear flow
}

\author{
T. Wu and C.-F. Chen, Taipei, Taiwan
}

(Received August 16, 1999)

Summary. By appealing to the classical boundary-layer theory, the present paper investigates the effect of the freestream shear on the separation of the laminar boundary layer around a circular cylinder. It is shown that on the side of the cylinder with faster freestream velocity the location of the separation point (point of vanishing wall shear) is virtually unaffected by the freestream shear, while on the other side of the cylinder a critical shear rate is observed. Below this critical value, separation occurs typically at the rear surface of the cylinder and is found to shift towards the downstream direction with increasing freestream shear. Above the critical shear rate, the boundary layer separates from the windward side of the cylinder. Further increase of the freestream shear then causes the separation point to move towards the upstream direction. The present findings may have important implication on the issue regarding to the orientation of the lift force exerting on the cylinder.

\section{Initroduction}

Ever since Prandtl first proposed the concepts of "boundary layer" and "flow separation" in his truly epoch-making paper [1] in 1904, the boundary-layer theory has played an important role in the development of modern fluid dynamics. Mathematically, the theory leads to a great simplification of the complete Navier-Stokes equations, and physically it describes the limiting behavior of the flow with infinite Reynolds number. It is generally accepted that the boundary-layer formulation is a valid approximation to large-Reynolds-number-flow until "separation" starts to intervene. In steady flows, such a feature is associated with the appearance of a vanishing wall shear and was described by Prandtl in his theory as flow "breaking away" from the surface. Early efforts in studying flow separation were then made by tracking the point of zero wall shear (see, e.g., Schlichting [2]) as it signals the onset of flow reversal and termination of the boundary-layer type flow.

Among all the benchmark studies in fluid mechanics, the problem of a uniform flow past a circular cylinder has long been a paradigm for exploring flow separation and related phenomena [2], [3]. The abundant and intriguing flow dynamics associated with yet such a simple configuration has attracted the attention of many researchers over a time span of nearly a century. Though with the advent of high-speed computers and delicate experimental techniques complicated flow phenomena about a circular cylinder have been disclosed and established to an extent far beyond the pertinent realm of the boudnary-layer theory, the fruitful achievements and the great success we enjoy today can be attributed to Prandtl's invaluable contribution. 
Interestingly, the more general case of flow separation over a circular cylinder placed in a uniform shear flow, which is of both theoretical and practical importance, appears to have received less attention. In practice, shear flow is a more commonly encountered one than the uniform stream; e.g., the atmospheric flow near the surface of the earth, the flow near the wall of a conduit with large cross-section, etc. There exist in literature some important results [4]-[7], mostly for low-Reynolds-number-flow, discussing the characteristic of the lift force acting on the circular cylinder due to the asymmetry of the shear flow. Some other studies [8] - [12], either numerical or experimental, are focussing on the frequency of the vortex shedding and the associated drag force over the cylinder in a linear shear flow. However, despite of the historical significance of the boundary-layer hypothesis and its simplicity in formulation, a fundamental study about the dependence of the separation location on the freestream shear as based on Prandtl's simple criterion of separation seems to be lacking. The present paper is intended to provide such a result.

In strict mathematical terms, the viscous boundary layer and the limiting inviscid-flow formulations construct the so called leading order inner and outer expansions, respectively, of the full Navier-Stokes equations. In the essence of the matched asymptotic expansions technique, the boundary-layer flow is then completely determined by the streamwise pressure gradient incurred from the outer inviscid flow. The outer flow can be either irrotational or with constant vorticity, as long as it satisfies the inviscid-flow equations. In the classical uniform stream past a circular cylinder problem, the outer inviscid flow is easily obtained from the well known complex potential theory. Probably less known is the inviscid solution of a uniform shear flow past a cylinder. Fortunately, as will be described in the next section, there is a nice theory (Milne-Thomson [13]) with which an exact analytical result for the circular cylinder case can be derived. With the inviscid flow distribution along the cylinder surface at hand, the flow in the boundary layer can then be readily solved via a conventional numerical scheme, and the separation is determined by locating the point where the wall shear vanishes.

Though it is well recognized that due to the negligence of the viscous-inviscid interaction existing in a finite-Reynolds-number-flow and the effect of the wake behind the cylinder the actual flow diverts somewhat from the classical boundary-layer result, the present work may nevertheless serve as a benchmark case for reference in fluid dynamics texts. It also provides a basic result upon which future improvement may be built up, such as the incorporation of a strong viscous-inviscid interaction model to attain a more realistic approximation to the flow.

\section{Formulations of the problem}

\subsection{Boundary-layer equations}

Let the velocity distribution of the freestream with uniform shear be given by

$U=U_{o}+\sigma y_{c}$

where $\sigma=\partial U / \partial y_{c}=-\zeta$ denotes the constant shear rate of the freestream and is equivalent by definition to the negative vorticity $\zeta$ of the flow, and $U_{0}$ is some reference velocity along a line passing through the center of the circular cylinder (see Fig. 1). According to Eq. (1), positive $\sigma$ refers to the freestream speed increasing linearly in the positive (upward) $y_{c}$-direction. The formulation is then given by the usual steady, incompressible two-dimensional boundary- 


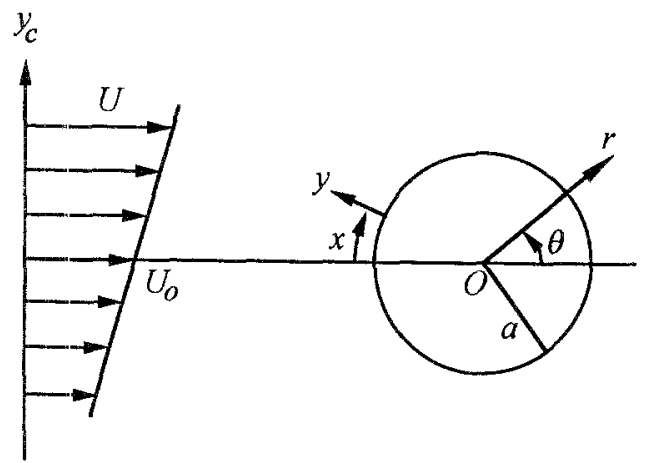

Fig. 1. Flow configuration and coordinate systems

layer equations:

$\frac{\partial u}{\partial x}+\frac{\partial v}{\partial y}=0$

$u \frac{\partial u}{\partial x}+v \frac{\partial u}{\partial y}=U_{e} \frac{d U_{e}}{d x}+\frac{\partial^{2} u}{\partial y^{2}}$,

in which $x$ and $y$ represent the boundary-layer coordinates along and normal to the cylinder surface, $u$ and $v$ are the velocity components along $x$ and $y$, respectively. As usual, Eqs. (2) and (3) have been normalized by the radius of the circular cylinder $a$ and the reference velocity $U_{o}$. Also, both the normal coordinate $y$ and velocity component $v$ have been scaled up by the Reynolds number $R_{e}=U_{o} a / \nu$. The above equations are subject to a no-slip condition on the cylinder surface, and an inviscid velocity distribution $U_{e}$ at the far field:

$u=v=0 \quad$ at $\quad y=0$,

$u \rightarrow U_{e} \quad$ as $\quad y \rightarrow \infty$.

The distribution of $U_{e}$ is to be obtained from the inviscid solution of the flow (derived in the next section) along the cylinder surface. The streamwise pressure gradient $d p / d x$ driving the boundary layer is then related to the velocity $U_{e}$ as $d p / d x=-U_{e} d U_{e} / d x$.

\subsection{Outer inviscid-flow solution}

The inviscid-flow solution to the problem can be obtained by using the second circle theorem [13] described as follows.

Let the stream function $\psi_{o}$ of an unperturbed (that is, without the presence of a cylinder or any solid boundary) two-dimensional flow with constant vorticity $\zeta_{o}$ be expressed as

$\psi_{o}(z, \bar{z})=F(z)+\bar{F}(\bar{z})-\frac{1}{4} \zeta_{o} z \bar{z}$,

where $z=x_{c}+i y_{c}, \bar{z}=x_{c}-i y_{c}$ is a pair of complex coordinates, $F(z)$ is a complex function, and overbarred quantities denote the complex conjugates of the associated function or variable. It is a trivial task to confirm that the vorticity of the flow represented by (5) is a constant:

$\zeta=-\left(\frac{\partial^{2} \psi_{o}}{\partial x_{c}{ }^{2}}+\frac{\partial^{2} \psi_{o}}{\partial y_{c}{ }^{2}}\right)=-4 \frac{\partial^{2} \psi_{o}}{\partial z \partial \bar{z}}=\zeta_{o}$. 
The second circle theorem states that when introducing a circular cylinder of radius $a$ (centered at the origin of the complex plane $z$ ) into the above base flow, the stream function of the resulting flow field is then given by

$\psi(z, \bar{z})=F(z)-F\left(\frac{a^{2}}{\bar{z}}\right)+\bar{F}(\bar{z})-\bar{F}\left(\frac{a^{2}}{z}\right)-\frac{1}{4} \zeta_{\circ} z \bar{z}$.

It is easy to show that along the circle $|z|=a$ the above stream function reduces to a constant $\psi=-1 / 4 \zeta_{o} a^{2}$, indicating that the circle is indeed a streamline. Far from the cylinder $z \rightarrow \infty$, Eq. (6) converges to the base flow (5). The last term in (6) accounts for the vorticity persistent to the flow. Again, the vorticity is a constant and equal to that of the base flow $\zeta_{o}$, in consistence with Kelvin's theorem [13] which states that the circulation in an inviscid, incompressible flow subject to conservative external forces is conserved. Thus the expression (6) is indeed the solution we are seeking, and the whole problem is nailed down to the determination of a proper function $F(z)$.

In our present problem, the unperturbed base flow is a free stream with constant vorticity $\zeta_{o}=-\sigma$ as given by (1), the corresponding stream function is, upon using the coordinates relation $y_{c}=-i / 2(z-\bar{z})$,

$\psi_{o}=U_{o} y_{c}+\frac{\sigma}{2} y_{c}{ }^{2}=\frac{-i U_{o}}{2}(z-\bar{z})-\frac{\sigma}{8}(z-\bar{z})^{2}=\frac{-i U_{o}}{2} z+\frac{i U_{o}}{2} \bar{z}-\frac{\sigma}{8} z^{2}-\frac{\sigma}{8} \bar{z}^{2}+\frac{\sigma}{4} z \bar{z}$

Comparing with Eq. (5), it is apparent that

$F(z)=\frac{-i U_{0}}{2} z-\frac{\sigma}{8} z^{2}$

Substituting $F(z)$ into Eq. (6) and taking the proper conjugate operations, we obtain.

$\psi(z, \bar{z})=\frac{-i U_{o}}{2} z-\frac{\sigma}{8} z^{2}+\frac{i U_{o} a^{2}}{2 \bar{z}}+\frac{\sigma a^{4}}{8 \bar{z}^{2}}+\frac{i U_{o}}{2} \bar{z}-\frac{\sigma}{8} \bar{z}^{2}-\frac{i U_{o} a^{2}}{2 z}+\frac{\sigma a^{4}}{8 z^{2}}+\frac{\sigma}{4} z \bar{z}$.

Written in terms of the polar coordinates $z=r e^{i \theta}$, the above expression becomes

$\psi(r, \theta)=U_{o}\left(r-\frac{a^{2}}{r}\right) \sin \theta-\frac{\sigma}{4}\left[\left(r^{2}-\frac{a^{4}}{r^{2}}\right) \cos 2 \theta-\left(r^{2}-a^{2}\right)\right]$.

In Eq. (8), a constant $-\sigma a^{2} / 4$ has been added to render the stream function $\psi=0$ along the circle $r=a$. The inviscid velocity along the cylinder surface can then be obtained by evaluating the derivative of the stream function (8) at $r=a$,

$V_{\theta}=-\left.\frac{\partial \psi}{\partial r}\right|_{r=a}=-2 U_{o} \sin \theta+\sigma a \cos 2 \theta-\frac{\sigma a}{2}$.

When normalized by the characteristic length $a$ and the reference velocity $U_{o}$, the nondimensional forms of (8) and (9) read

$\psi(r, \theta)=\left(r-\frac{1}{r}\right) \sin \theta-\frac{\sigma}{4}\left[\left(r^{2}-\frac{1}{r^{2}}\right) \cos 2 \theta-\left(r^{2}-1\right)\right]$,

$V_{\theta}=-2 \sin \theta+\sigma \cos 2 \theta-\frac{\sigma}{2}$,

with $\sigma$ now denoting the dimensionless shear rate $\left(\sigma=-\zeta_{0} a / U_{o}\right)$ of the free stream. 
Finally, in conformity with the direction of the dimensionless boundary-layer coordinate $x$ defined in Fig. 1, the inviscid velocity distribution $U_{e}$ along the circular cylinder is re-written as (in nondimensional form):

$U_{e}=-V_{\theta}=2 \sin x-\sigma \cos 2 x+\frac{\sigma}{2}$.

Equation (12) serves as the boundary condition at the outer edge of the boundary layer.

\subsection{Stagnation points and stagnation flow}

According to Eq. (12), the location of the stagnation point $x_{o}$ (where $U_{e}=0$ ) on the cylinder surface is given by

$x_{o}=\sin ^{-1}\left(\frac{-1 \pm \sqrt{1+\sigma^{2}}}{2 \sigma}\right)$.

The above expression is graphed in Fig. 2, because of symmetry only the variation of the stagnation location at the windward side (front stagnation) is shown. Depending on the value of $\sigma$, there may be two stagnation points (one at front and the other at rear) on the cylinder for $-4 / 3<\sigma<4 / 3$, or four stagnation points otherwise. The presence of the extra two stagnation points is owing to the fact that for large $|\sigma|$ value one side of the cylinder surface is covered with flow that travels in the opposite direction. To show this, inviscid streamlines (10) are plotted for three different values of $\sigma$ in Fig. 3. At $\sigma=1$, only two stagnation points are present on the cylinder, the other stagnation point, appearing as a saddle in the plot, lies within the flow. At a critical value $\sigma=4 / 3$, the saddle point attaches to the cylinder surface. It then splits into two stagnation points, separating apart along the cylinder surface with increasing $\sigma$. In the latter case, a portion of the cylinder surface in the lower half is covered with a back flow. To simplify the issue, the boundary-layer calculation and discussion made in this paper will be restricted to the parameter range $-4 / 3<\sigma<4 / 3$ only. It is also observed in Fig. 3 that within this range the location of the front stagnation point is biased towards the fast side of the freestream as noted in [6].

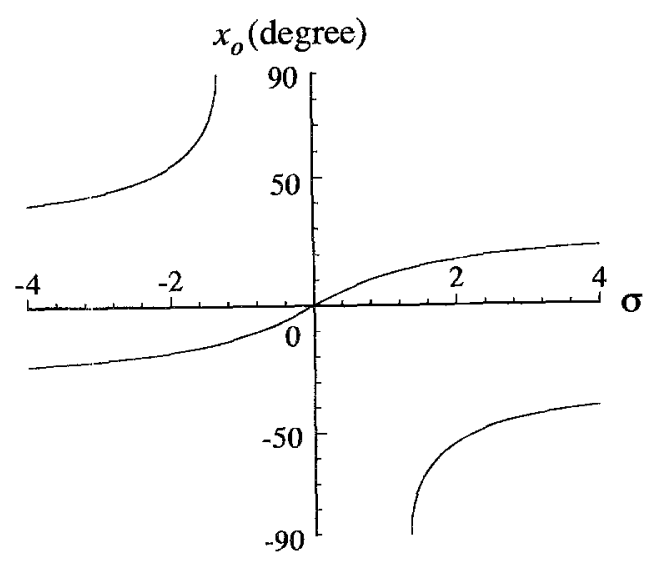

Fig. 2. Variation of front stagnation point $x_{0}$ with freestream shear rate $\sigma$ 


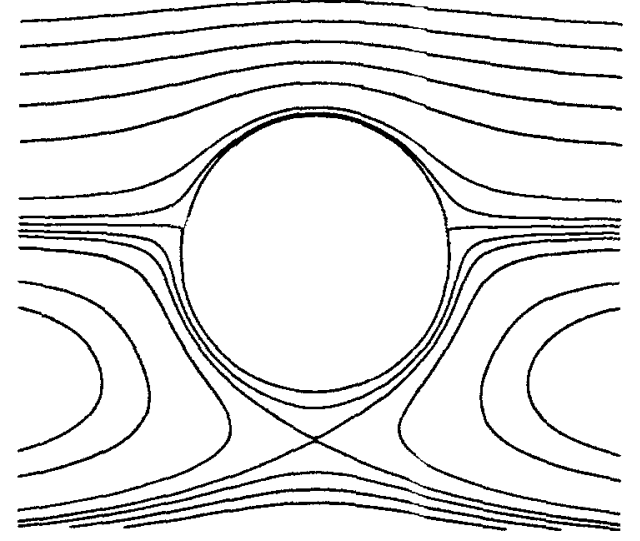

a

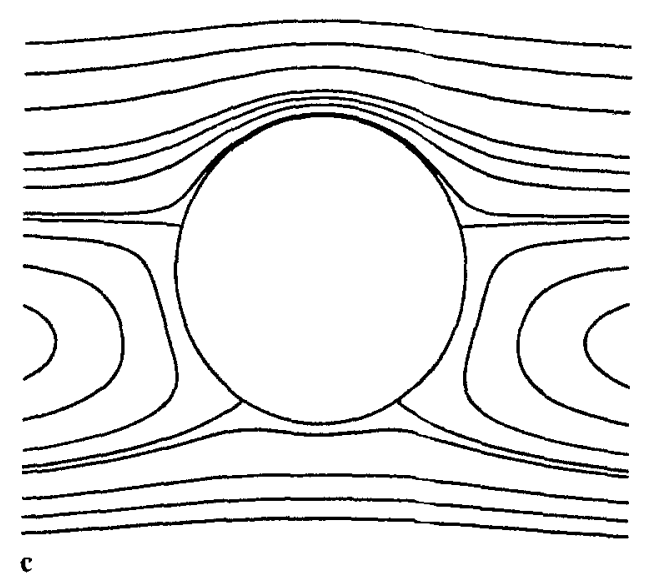

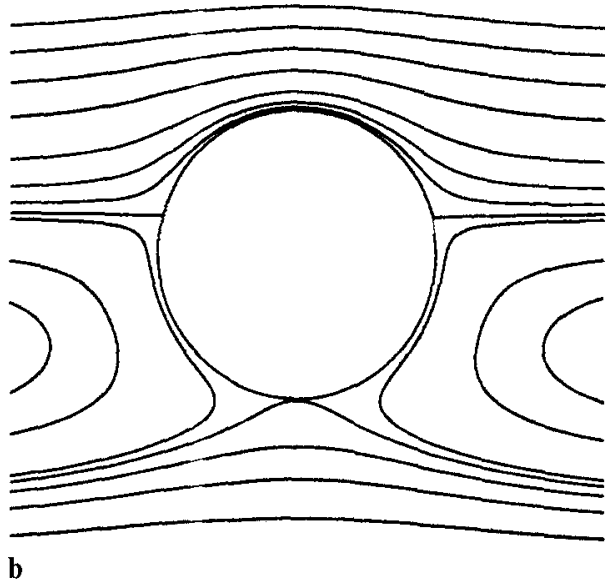

Fig. 3. Inviscid streamlines around the circular cylinder under three different freestream shear rates: $\mathbf{a} \sigma=1.0, \mathbf{b} \sigma=4 / 3, \mathbf{c} \sigma=1.8$.

The inviscid velocity near the front stagnation point $x_{o}$ can be derived from expanding Eq. (12) for small $x-x_{0}$ :

$U_{e}(x) \cong 0+\left.\frac{d U_{e}}{d x}\right|_{x_{o}}\left(x-x_{o}\right)=\left(2 \cos x_{o}+2 \sigma \sin 2 x_{o}\right)\left(x-x_{o}\right)$.

Equation (13) gives a linear distribution of $U_{e}$ in $\left(x-x_{o}\right)$. Furthermore, from the inviscid solution (10), it is not hard to show that the stagnation streamline intersects the cylinder surface perpendicularly at the stagnation point. Thus disregarding the presence of a simple shear in the freestream, locally the boundary layer in the neighborhood of the stagnation point can still be treated as the well known plane stagnation flow of Hiemenz. The governing equation for the Hiemenz flow is [2]

$\frac{d^{3} f}{d \eta^{3}}+f \frac{d^{2} f}{d \eta^{2}}-\left(\frac{d f}{d \eta}\right)^{2}+1=0$

with

$\eta=y \sqrt{\left.\frac{d U_{e}}{d x}\right|_{x_{0}}}, \quad \frac{d f}{d \eta}=\left.\frac{u}{U_{e}}\right|_{x_{0}}$.

The equation and its solution are well stated in most of the texts (e.g., see Schlichting [2]). The solution is then used as the initial condition for starting the numerical integration of the viscous boundary-layer equations in the next section. 


\section{Numerical method}

The streamwise velocity $u$ along the stagnation line in the viscous boundary layer is identically zero. In order to employ the Hiemenz solution as the starting velocity profile for integrating the flow downstream, the boundary-layer equations (2) and (3) need to be modified slightly. Let us define "reduced" velocities as

$u=s \tilde{u}, \quad U_{e}=s \tilde{U}_{e}$,

with $s=x-x_{0}$ denoting the distance to the front stagnation point $x_{0}$, then the governing equations (2), (3) become (in conservative form)

$\frac{\partial(s \tilde{u})}{\partial x}=-\frac{\partial v}{\partial y}$

$\frac{1}{2} \frac{\partial\left(s \tilde{u}^{2}\right)}{\partial x}-\frac{1}{2} \frac{\partial\left(s \tilde{U}_{e}^{2}\right)}{\partial x}=\frac{\partial^{2} \tilde{u}}{\partial y^{2}}-v \frac{\partial \tilde{u}}{\partial y}+\frac{1}{2}\left(\tilde{U}_{e}^{2}-\tilde{u}^{2}\right)$.

Note that the flow variable in the Hiemenz equations (14), (15) is related to the newly defined reduced velocities by $d f / d \eta=\left(\tilde{u} / \tilde{U}_{e}\right)_{s=0}$. Therefore, in what follows we are actually solving for the reduced velocity $\tilde{u}$ from Eqs. (16) and (17).

In the calculation, the unbounded physical domain $0 \leq y \leq \infty$ is more conveniently transformed to a unit computational region $0 \leq \beta \leq 1$ by the mapping function

$y=A \cdot \tan \left(\frac{\pi}{2} \beta\right)$

where $A$ is a mapping constant controlling the grid spacing near the cylinder surface. In terms of $\beta$, Eqs. (16) and (17) become

$\frac{\partial(s \tilde{u})}{\partial x}=-\beta, y \frac{\partial v}{\partial \beta}$,

$\frac{1}{2} \frac{\partial\left(s \tilde{u}^{2}\right)}{\partial x}-\frac{1}{2} \frac{\partial\left(s \tilde{U}_{e}^{2}\right)}{\partial x}=\beta,{ }_{y}^{2} \frac{\partial^{2} \tilde{u}}{\partial \beta^{2}}+(\beta, y y-v \beta, y) \frac{\partial \tilde{u}}{\partial \beta}+\frac{1}{2}\left(\tilde{U}_{e}^{2}-\tilde{u}^{2}\right)$

in which $\beta, y$ and $\beta, y y$ are shorthand notations for the first and second derivatives of the mapping function. The proper boundary conditions for the above equations are then

$\tilde{u}=v=0 \quad$ at $\beta=0$,

$\tilde{u}=\tilde{U}_{e} \quad$ at $\beta=1$.

A uniform grid is adopted in the $\beta$ direction, and Eqs. (18), (19) are discretized in terms of the computational coordinates $(x, \beta)$.

The numerical scheme used to integrate Eqs. (18) and (19) is the conventional spacemarching technique initiated from the front stagnation line with the Hiemenz velocity profile

$\tilde{u}=\tilde{U}_{e} \frac{d f}{d \eta} \quad$ at $\quad s=0$.

The method is standard (see the text by Anderson [14]), and the procedure is described briefly as follows.

All $\beta$-derivative terms are approximated by central differences, and a second-order CrankNicolson scheme is applied to the streamwise derivatives. Introducing incremental variables

$$
(d \tilde{u})_{i+1, j}=\tilde{u}_{i+1, j}-\tilde{u}_{i, j}, \quad(d v)_{i+1, j}=v_{i+1, j}-v_{i, j}, \quad\left(d \tilde{U}_{e}\right)_{i+1}=\tilde{U}_{e_{i+1}}-\tilde{U}_{e_{i}},
$$


where indices $i, j$ denote the grid levels in the $x$ - and $\beta$-coordinates, the resulting finite-difference equation for (19), after re-arrangement, can be put into a form with tridiagonal coefficient matrix:

$C_{j}(d \tilde{u})_{i+1, j-1}+D_{j}(d \tilde{u})_{i+1, j}+E_{j}(d \tilde{u})_{i+1, j+1}=S_{i+1, j}$,

where

$$
\begin{aligned}
& C_{j}=\frac{\left(\beta,_{y}^{2}\right)_{j}}{2(\Delta \beta)^{2}}-\frac{1}{4 \Delta \beta}\left[\left(\beta, y_{y y}\right)_{j}-(\beta, y)_{j} v_{i, j}\right], \\
& D_{j}=-\frac{\left(\beta,_{y}^{2}\right)_{j}}{(\Delta \beta)^{2}}-\left(\frac{1}{2}+\frac{s_{i+1}}{\Delta x}\right) \tilde{u}_{i, j}, \\
& E_{j}=\frac{\left(\beta,_{y}^{2}\right)_{j}}{2(\Delta \beta)^{2}}+\frac{1}{4 \Delta \beta}\left[(\beta, y y)_{j}-(\beta, y)_{j} v_{i, j}\right],
\end{aligned}
$$

and the source term is

$$
\begin{aligned}
S_{i+1, j}= & -\left\{\frac{\left.(\beta)_{y}^{2}\right)_{j}}{(\Delta \beta)^{2}}-\frac{1}{2 \Delta \beta}\left[(\beta, y y)_{j}-(\beta, y)_{j}\left(v_{i, j}+\frac{(d v)_{i+1, j}}{2}\right)\right]\right\} \tilde{u}_{i, j-1} \\
& -\left\{\frac{(\beta, y)_{j}}{(\Delta \beta)^{2}}+\frac{1}{2 \Delta \beta}\left[(\beta, y y)_{j}-(\beta, y)_{j}\left(v_{i, j}+\frac{(d v)_{i+1, j}}{2}\right)\right]\right\} \tilde{u}_{i, j+1} \\
& +\frac{2(\beta, y)_{j}}{(\Delta \beta)^{2}} \tilde{u}_{i, j}+\left(\frac{1}{4}+\frac{s_{i+1}}{2 \Delta x}\right)(d \tilde{u})_{i+1, j}^{2}+\frac{(\beta, y)_{j}}{4 \Delta \beta}(d v)_{i+1, j}\left((d \tilde{u})_{i+1, j}-(d \tilde{u})_{i-1, j}\right) \\
& +\frac{1}{2 \Delta x}\left[\Delta x\left(\tilde{u}_{i, j}\right)^{2}-\Delta x\left(\tilde{U}_{e_{i}}\right)^{2}-2 s_{i+1} \tilde{U}_{e_{i}}\left(d \tilde{U}_{e}\right)_{i+1}-s_{i+1}\left(d \tilde{U}_{e}\right)_{i+1}^{2}\right] \\
& -\frac{1}{2}\left(\tilde{U}_{e_{i}}^{2}-\tilde{u}_{i, j}^{2}\right)-\frac{1}{4}\left[2 \tilde{U}_{e_{i}}\left(d \tilde{U}_{e}\right)_{i+1}+\left(d \tilde{U}_{e}\right)_{i+1}^{2}\right]
\end{aligned}
$$

Notice that we have retained all the high-order terms like $(d \tilde{u})^{2},(d v)(d \tilde{u})$ in the source term $S$ for accuracy considerations. Due to the presence of these nonlinear terms, Eq. (22) must be solved iteratively. Using an efficient tri-diagonal matrix solver [15], Eq. (22) can be easily solved for $(d \tilde{u})_{i+1, j}$ by first guessing a trial value for $(d v)_{i+1, j}$ and $(d \tilde{u})_{i+1, j}$ in the expression of $S_{i+1, j}$. Having obtained the reduced velocity $\tilde{u}_{i+1, j}$, the normal velocity $v_{i+1, j}$ can be computed from integrating the continuity equation

$\frac{1}{2}\left(\beta_{y y}\right)_{j}\left(\frac{\partial v}{\partial \beta}\right)_{i+1, j}=-\frac{1}{2}\left(\beta_{, y}\right\}_{j}\left(\frac{\partial v}{\partial \beta}\right)_{i, j}-\frac{s_{i+1} \tilde{u}_{i+1, j}-s_{i} \bar{u}_{i, j}}{\Delta x}$

by the trapezoidal rule. The values are then substituted back into the source term $S_{i+1, j}$ in (22) to calculate a new and improved $(d \tilde{u})_{i+1, j}$. The process is repeated until the deviations between two successive iterates of $\tilde{u}_{i+1, j}$ and $v_{i+1, j}$ are both smaller than a specified tolerance, then the solution is advanced to the next streamwise location $i+2$. Normally, 20 iterations are sufficient to bring the error down to $10^{-10}$. The number of iterations increases as the separation draws near. In the present work, the solution was first advanced with a uniform step size $\Delta x$. Close to separation, the step size was successively halved in order to obtain a better estimation of the separation location. Due to the presence of the freestream shear, the boundary layer develops asymmetrically along the upper and lower surfaces of the cylinder. Calculations 
were then performed separately, starting from the stagnation line, for the upper and lower boundary layers under different values of $\sigma$.

We believe that by retaining the nonlinear terms in the discretized equation (22) and utilizing Hiemenz' exact solution as the starting velocity profile along the front stagnation line, the present numerical scheme can generate a more accurate solution than other existing schemes.

\section{Results and discussion}

Since the free stream with a negative shear rate is just the up-side-down case of that with positive $\sigma$, only the results calculated for positive $\sigma$ will be presented here. Before showing the results, the accuracy of the aforementioned numerical scheme is assessed by examining the effect of grid refinement on the solution of the classical problem of a uniform flow $(\sigma=0)$ past a circular cylinder. Table 1 lists the location of separation $x_{s}$ computed on three successively finer meshes, $129 \times 0.01,257 \times 0.005$, and $513 \times 0.0025$. The two numerals associated with the mesh denote the number of grid points and the step size in the $\beta$ and $x$ directions, respectively. The results calculated over the three different grid settings appear to be all in good agreement with the reported value of $104.5 \mathrm{deg}$ in [2]. Figure 4 shows another grid refinement test on the wall shear distribution $\tau_{w}$ near separation calculated with nonzero free stream shear $(\sigma=1)$. Quadratic convergence of the numerical solution is affirmative in this plot. Also, it is observed from the figure that at separation the wall shear drops to zero with a singular slope, in consistence with the theory of Goldstein [16]. Unless noted otherwise, the following results are all based on calculations with the finest grid, $513 \times 0.0025$.

Variation of the separation location $x_{s}$ with freestream shear $\sigma$ is shown in Fig. 5. For the boundary layer along the upper half of the cylinder (i.e., boundary layer on the faster free strearn side), the separation point shifts only slightly towards the upstream direction with increasing $\sigma$ (from $104.5 \mathrm{deg}$ at $\sigma=0$ to $100.7 \mathrm{deg}$ at $\sigma=4 / 3$ ). However, a drastically different picture emerges for the boundary layer at the lower half of the cylinder. Here, increasing

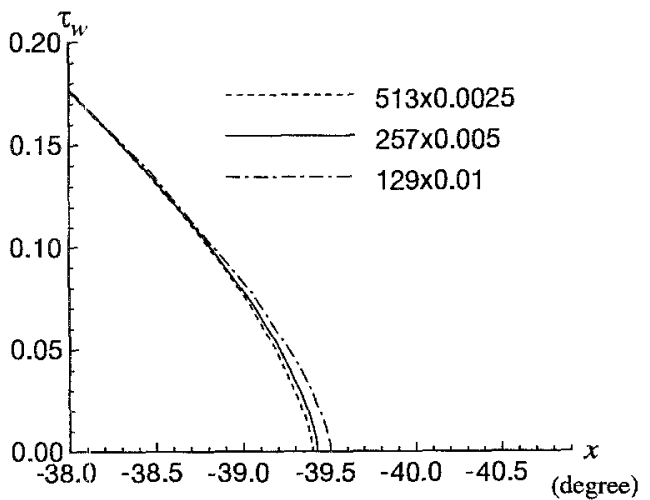

Fig. 4. Comparison of wall shear distributions $\tau_{w}$ calculated over three different mesh sizes, $\sigma=1.0$

Table 1. Separation location $x_{s}$ calculated over three different mesh sizes, $\sigma=0$

\begin{tabular}{ll}
\hline Mesh size & $x_{s}($ deg $)$ \\
\hline $129 \times 0.01$ & 104.656 \\
$257 \times 0.005$ & 104.567 \\
$513 \times 0.0025$ & 104.533 \\
\hline
\end{tabular}




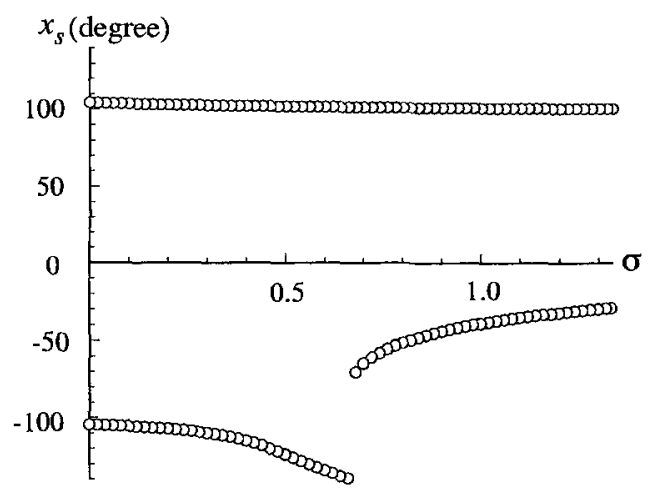

Fig. 5. Variation of separation location $x_{s}$ with freestream shear rate $\sigma$

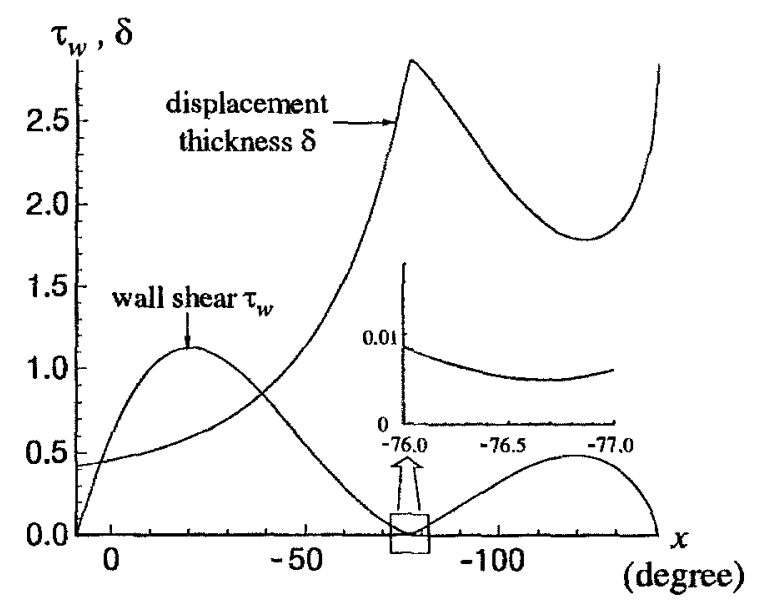

Fig. 6. Wall shear $\tau_{w}$ and displacement thickness distribution $\delta$ along the lower half of the cylinder at critical shear rate $\sigma_{c}=0.672$

the freestream shear delays the separation at first. Then, beyond a certain critical value of the shear rate $\sigma_{c}$, the separation point suddenly "jumps" forward to the windward side of the cylinder and continues to move upstream with increasing $\sigma$. Detailed computation shows that this critical value occurs at $\sigma_{c}=0.672$. It is then interesting to learn how the boundary layer behaves at this critical stage.

Distributions of the wall shear $\tau_{w}$ and the displacement thickness $\delta$ of the boundary layer along the lower half of the cylinder at $\sigma=0.672$ are given in Fig. 6. It is observed that at this critical shear rate the wall shear reaches a local minimum value (but still slightly above zero) at $x=-76.67 \mathrm{deg}$. After that, it increases again and finally falls down to zero singularly at a farther location $x=-140.4 \mathrm{deg}$. The distribution of the displacement thickness shows a similar feature. Though the actual separation occurs at $x=-140.4 \mathrm{deg}$, it is evident that the boundary layer has a strong tendency to develop a second separation in the upstream site. Indeed, at the shear rate slightly larger than 0.672 , separation first appears at a windward side location $x=-70.46 \mathrm{deg}$. In constrast to the case where the freestream shear is below the critical value, separation now moves towards the upstream direction with increasing $\sigma$ (e.g., $x_{s}=-29.43 \mathrm{deg}$ at $\sigma=4 / 3$ ).

The situation can be fully understood by looking at the inviscid streamlines near the cylinder surface (Fig. 3) and the associated pressure gradient $d p / d x$ imposed on the boundary layer (Fig. 7). Increasing the freestream shear forces the third stagnation point (the saddle) originally resides in the interior of the flow to move closer (along $\theta=270 \mathrm{deg}$ ) to the cylinder 


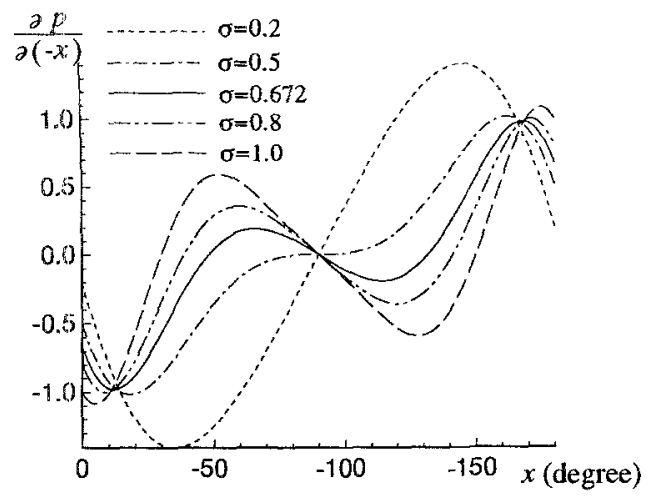

Fig. 7. Streamwise pressure gradient disributions along the lower half of the cylinder under different freestream shears

surface. Thus, the inviscid flow on the windward side of the cylinder is retarded, and an "adverse" streamwise pressure gradient is gradually formed in this area. As shown in Fig. 7, roughly beyond $\sigma=0.5$, a region with positive pressure gradient (positive when advancing in the downstream direction) appears at the windward side of the cylinder. On account of the fact that a boundary layer cannot withstand even a mildly adverse pressure gradient, it then separates immediately from the windward side. Further increasing $\sigma$ results in a "more adverse" pressure gradient and hence promotes the separation at a location farther upstream.

The consequence is therefore an interesting one. For small freestream shear, the result in Fig. 5 suggests that the boundary layer along the lower surface separates at a location farther downstream than that along the upper surface. If not considering the turbulence effect, the lift force acting on the cylinder will be most likely pointing downward in this case, i.e., towards the side with slower freestream velocity. However, for larger freestream shear, the separation of the lower boundary layer is advanced to the windward side, and the lift force may now act in the opposite direction. This aspect might explain the perplexing result reported in [7] where the lift coefficient was found to change sign with the shear parameter. In real flows, of course, the situation is further complicated by the issues of instability of the laminar boundary layer and the effect of turbulence re-attachment. The above observation has not been discussed in other previous investigations ([6], [9]-[12]), probably because the ranges of the shear rate $\sigma$ quoted in their studies are too small to capture the phenomenon. Also, the Reynolds numbers assumed by them are far too low to define a meaningful boundary layer. Nevertheless, the present study brings up a fundamental and yet potentially important issue awaiting for more elaborate analysis.

\section{Conclusion}

In the present paper, the boundary layer over a circular cylinder placed in a uniform shear flow has been studied in the framework of the classical boundary-layer theory. Specifically, the variation of the separation location (point of zero wall shear) under different freestream shears has been determined numerically for a range of the shear parameter $0 \leq \sigma \leq 4 / 3$ by integrating the steady boundary-layer equations via a proposed numerical scheme. Calculated results show that on the side of the cylinder with faster freestream velocity the location of the separation is not significantly affected by the magnitude of the freestream shear, while on the other side of the cylinder increasing the freestream shear results in a downstream shifting of 
the separation point at first. At the critical shear rate $\sigma_{c}=0.672$, the boundary layer starts to separate from the windward side of the cylinder instead. A further increase of the freestream shear then causes the separation point to move in the upstream direction. Thus, depending on the magnitude of the freestream shear, the separation of the boundary layer may have opposite effects on the orientation of the lift force acting on the cylinder.

The present work extends the classical boundary-layer result to a more general case involving a constant shear in the freestream. The critical role played by the shear parameter $\sigma$ in delaying/provoking a separation has been established on a theoretical basis. The formulation of the problem considered here is rather idealized, yet more realistic results can always be achieved by modifying the outer inviscid velocity with that from a viscous-inviscid interaction model or from experimental measurements. In practice, many interesting flows can be simulated or approximated by a linear shear flow, the repercussion of the underlying feature demonstrated in this study is then a far-reaching one.

\section{References}

[1] Prandtl, L.: Über Flüssigkeitsbewegung bei sehr kleiner Reibung. In: Proc. 3rd Int. Math. Congress, pp. 484-491. Heidelberg 1904.

[2] Schlichting, H.: Boundary-layer theory, 7th ed. New York: McGraw-Hill 1979.

[3] Zdravkovich, M. M.: Flow around circular cylinders. Oxford: Oxford University Press 1996.

[4] Bretherton, F. P.: Slow viscous motion round a cylinder in a simple shear. J. Fluid Mech. 12, $591-613$ (1962).

[5] Saffman, P. G.: The lift on a small sphere in a slow shear flow. J. Fluid Mech. 22, 385-400 (1965).

[6] Jordan, S. K., Fromm, J. E.: Laminar flow past a circle in a shear flow. Phys. Fluids 15, 972-976 (1972).

[7] Nakabayashi, K., Yoshida, N., Aoi, T.: Numerical analysis for viscous shear flows past a circular cylinder at intermediate Reynolds numbers. JSME Int. J. Ser. B 36, 34-41 (1993).

[8] Maull, D. J., Young, R. A.: Vortex shedding from bluff bodies in a shear flow. J. Fluid Mech. 60, 401-409 (1973).

[9] Kossack, C. A., Acrivos, A.: Steady simple shear flow past a circular cylinder at moderate Reynolds numbers: a numerical solution. J. Fluid Mech. 66, 353-376 (1974).

[10] Kiya, M., Tamura, H., Arie, M.: Vortex shedding from a circular cylinder in moderate Reynolds number shear flow. J. Fluid Mech. 101, $721-735$ (1980).

[11] Kwon, T. S., Sung, H. J., Hyun, J. M.: Experimental investigation of uniform-shear flow past a circular cylinder. Trans. ASME J. Fluids Eng. 114, 457-460 (1992).

[12] Chew, Y. T., Luo, S. C., Cheng, M.: Numerical study of a linear shear flow past a rotating cylinder. J. Wind Engineering and Industrial Aerodynamics 66, $107-125$ (1997).

[13] Milne-Thomson, L. M.: Theoretical aerodynamics, 4th ed., pp. 93-95. New York: Dover 1973.

[14] Anderson, J. D., Jr.: Computational fluid dynamics. New York: McGraw-Hill 1995.

[15] Press, W. H., Teukolsky, S. A., Vetterling, W. T., Flannery, B. P.: Numerical recipes, 2nd ed., pp. 42-43. Cambridge: Cambridge University Press 1992.

[16] Goldstein, S.: On laminar boundary layer flow near a position of separation. Q. J. Mech. Appl. Math. 1, $43-69$ (1948).

Authors' address: Prof. T. Wu and C.-F. Chen, Department of Mechanical Engineering, National Taiwan University, Taipei, Taiwan 